\title{
THE BEPPOSAX X-RAY VIEW OF REFLECTION-DOMINATED SEYFERT GALAXIES
}

\author{
M.Guainazzi ${ }^{1}$, G.Matt ${ }^{2}$ and F.Fiore ${ }^{3}$ \\ ${ }^{1}$ Astrophysics Division, Space Science Department of ESA, ESTEC/SA, Postbus 299, NL-2200 AG No- \\ ordwijk, The Netherlands \\ mguainaz@astro.estec.esa.nl \\ ${ }^{2}$ Dipartimento di Fisica "E.Amaldi", Università degli Studi "Roma Tre", Via della Vasca Navale 84, \\ I-00146 Roma, Italy \\ ${ }^{3}$ Osservatorio Astronomico di Roma, Via dell'Osservatorio, I-00144, Monteporzio Catone, Italy
}

\section{ABSTRACT}

We present new results from BeppoSAX observations of reflection-dominated Seyfert galaxies, and namely: 1) the Compton-thick Seyfert 2 NGC 1068 and Circinus Galaxy; 2) the Seyfert 1 NGC 4051, whose nucleus was observed on May 1998 to have switched off, leaving only a residual reflection component as an echo of its past activity. Our main focus in this paper is on the soft X-ray continuum properties and on the X-ray line spectroscopy.

\section{COMPTON-THICK SEYFERT 2 GALAXIES}

Despite the fact that Seyfert galaxies are classified according mainly to optical properties, the X-ray behavior of type 1 and 2 objects is remarkably different, the main difference laying in the amount of photoelectric absorption from neutral matter. Seyfert 2 s normally exhibit column densities $\mathrm{N}_{\mathrm{H}} \gtrsim 10^{21} \mathrm{~cm}^{-2}$ (Awaki et al. 1991; Turner at el. 1997), but in a few cases no significant column density is observed above the Galactic contribution. A key to understand this apparent oddity came from the discovery of broad optical lines in the polarized optical spectra of several Seyfert 2s (Antonucci \& Miller 1985; Tran 1995; Heisler et al. 1997), which posed the basis for the so called "Seyfert unification theories" (Antonucci 1993). In some "Compton-thick" objects, the interposing matter is optically thick to Compton scattering, and therefore the impinging photons are down-scattered to energies where the photoabsorption cross section becomes dominant. In these cases the primary nuclear continuum is invisible, unless scattered and/or reflected along our line of sight. X-rays might follow the same optical path as the optical photons, allowing view of the radiation produced in the innermost nuclear regions, close to the central black hole.

If this is indeed the case, one might expect that the scattering plasma ("warm mirror") adds a wealth of emission lines from ionized elements, due to fluorescence/recombination and/or resonant scattering. ASCA observations unveiled that the X-ray spectrum of Compton-thick Seyfert $2 \mathrm{~s}$ is indeed rich of emission lines, mainly due to He-like stages of intermediate elements (Matt et al. 1997b; Iwasawa et al. 1997; Turner et al. 1997). However, in all these cases the flat observed continuum (photon index $\Gamma<1$ ) and a huge fluorescent iron line from neutral matter (Equivalent Width $\mathrm{EW}>1 \mathrm{keV}$ ) suggest that the intermediate X-ray spectrum is dominated by Compton reflection from optically thick neutral matter (Matt et al. 1997b). Moreover, several Seyfert 2s host intense nuclear starbursts, which are expected 
Table 1: Emission lines in the BeppoSAX spectra of NGC 1068 and Circinus Galaxy

\begin{tabular}{|c|c|c|c|c|}
\hline \multirow[b]{2}{*}{ Identification } & \multicolumn{2}{|c|}{ NGC 1068} & \multicolumn{2}{|c|}{ Circinus Galaxy } \\
\hline & $\begin{array}{c}\mathrm{E}_{\mathrm{C}} \\
(\mathrm{keV})\end{array}$ & $\begin{array}{l}\text { EW } \\
(\mathrm{eV})\end{array}$ & $\begin{array}{c}\mathrm{E}_{\mathrm{C}} \\
(\mathrm{keV})\end{array}$ & $\begin{array}{l}\text { EW } \\
(\mathrm{eV})\end{array}$ \\
\hline OVII & $0.54 \pm 0.04$ & $310 \pm 140^{\star}$ & $\ldots$ & $\ldots$ \\
\hline NeIX & $0.95 \pm 0.03$ & $320 \pm 100^{\star}$ & $\ldots$ & $\ldots$ \\
\hline MgXI & $1.32 \pm 0.05$ & $170 \pm 70^{\star}$ & $1.32^{\dagger}$ & $<50^{\star}$ \\
\hline SixiII & $1.88 \pm 0.03$ & $280 \pm 60^{\star}$ & $1.87 \pm_{0.05}^{0.04}$ & $160 \pm_{60}^{80 \star}$ \\
\hline SxV & $2.45 \pm 0.04$ & $210 \pm 50^{\star}$ & $2.43 \pm_{0.04}^{0.05}$ & $260 \pm 70^{\star}$ \\
\hline ArXVII & $3.1^{\dagger}$ & $<5^{\star}$ & $3.14 \pm_{0.09}^{0.11}$ & $140 \pm_{80}^{60 \star}$ \\
\hline Iron (nickel?) lines: & & & & \\
\hline "Neutral" & $6.4^{\dagger}$ & $1600 \pm 200^{\diamond}$ & $6.446 \pm_{0.013}^{0.012}$ & $2850 \pm 130^{\diamond}$ \\
\hline "Neutral" $\left(\mathrm{K}_{\beta}\right)$ & $\ldots$ & $\ldots$ & $7.08 \pm 0.06$ & $640 \pm_{110}^{100 \diamond}$ \\
\hline Fexxv & $6.7^{\dagger}$ & $3000 \pm 600^{\star}$ & $6.7^{\dagger}$ & $<70^{\star}$ \\
\hline $\operatorname{Fexxv}\left(\mathrm{K}_{\beta}\right)\left(\right.$ or Ni $\left.\mathrm{K}_{\alpha}\right)$ & $\ldots$ & $\ldots$ & $7.9 \pm_{0.3}^{0.2}$ & $700 \pm 300^{\star}$ \\
\hline Fexxvi & $6.96^{\dagger}$ & $1500 \pm_{500 \star}^{400 \star}$ & $\ldots$ & $\ldots$ \\
\hline $\operatorname{FexXVI}\left(\mathrm{K}_{\beta}\right)$ & $8.1 \pm 0.2$ & $600 \pm 300^{\star}$ & $\ldots$ & $\ldots$ \\
\hline
\end{tabular}

${ }^{\dagger}$ fixed $\quad$ *against the scattering $\quad \diamond$ against the Compton reflection

to produce a not negligible contribution to the soft X-ray spectrum (Ptak et al. 1998). The spectra of Compton-thick Seyfert 2s are therefore complex and the limited ASCA energy bandpass strongly hampers an accurate and unique deconvolution, which is essential to evaluate the properties of the emission lines, in particular the intensity against their proper continuum.

The scientific payload on board BeppoSAX (Boella et al. 1997) covers the unprecedented wide energy interval between 0.1 and $200 \mathrm{keV}$, and hence allows to circumvent the problem encountered by ASCA. In this section, we will report the results of the observation of two close and bright Compton-thick galaxies observed during the AO1 phase: NGC 1068 and Circinus Galaxy. Both these galaxies have polarized broad optical lines (Antonucci \& Miller 1985; Oliva et al. 1998) and host intense nuclear starburst rings (Scoville 1988; Maiolino et al. 1998). Details on the data reduction can be found in Matt et al. (1997a). A more detailed analysis can be found in Guainazzi et al. (1999).

\subsection{Main Observational Results}

The hard X-ray (i.e.: $\gtrsim 4 \mathrm{keV}$ ) spectra of NGC 1068 and Circinus Galaxy exhibit a substantial amount of Compton-reflection (Matt et al. 1997a; Matt et al 1999), which dominates above several keV. The photon spectral index $\Gamma$ of the primary component is $\simeq 2.1$ in NGC 1068 and $\simeq 1.6$ in Circinus Galaxy. These values are close to those observed in Seyfert 1 galaxies (Nandra et al. 1997), as foreseen by the unified theories. In Circinus Galaxy a prominent excess in the PDS band (i.e.: $\gtrsim 10 \mathrm{keV}$ ) can be most easily explained as due to the transmission of the same primary continuum through an absorbing screen of $\simeq 4 \times 10^{24} \mathrm{~cm}^{-2}$. The case of Circinus is particularly intriguing, since a cutoff in the primary continuum is required $\left(\mathrm{E}_{\text {cutoff }} \simeq 60 \mathrm{keV}\right)$. This is the first time that such a cutoff is detected in a Seyfert 2 .

A prominent soft excess is present in both sources, along with a rich set of emission lines (see Table 1). The soft excess can be only partly accounted for by scattering of the primary continuum, and cannot be modeled by a multi-temperature optically thin emission, as suggested by Ueno et al. (1994) The 

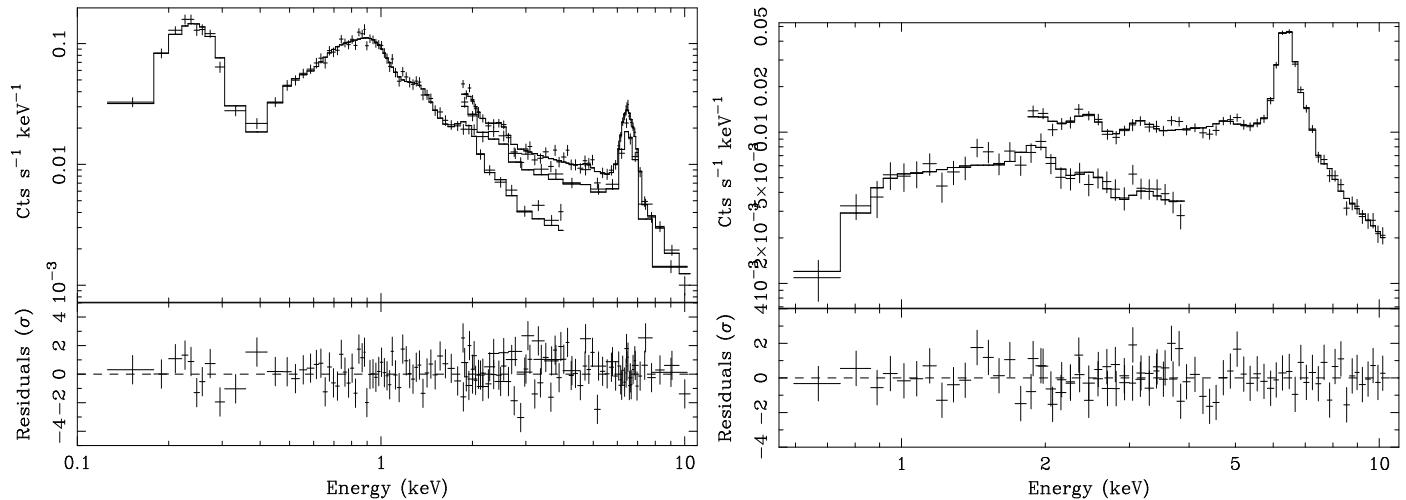

Figure 1: Spectra and residuals in units of standard deviations when the best fit model is applied to NGC 1068 (left) and Circinus Galaxy (right). The best fit model is composed of: a bare Comptonreflection continuum, a scattering component and a single temperature optically thin plasma emission (model MEKAL in XSPEC). Best-fit parameters are reported in Table 2
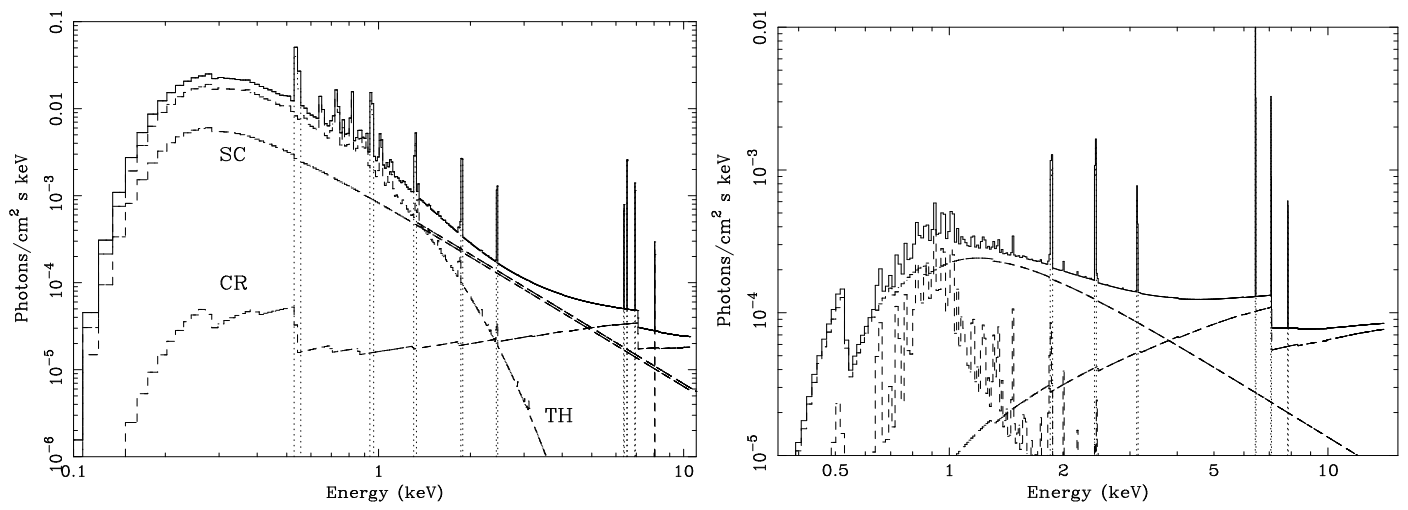

Figure 2: Best-fit models for NGC 1068 (left) and Circinus Galaxy (right). The continuum spectra components are labeled: CR (Compton reflection); SC (scattering), TH (thermal plasma)

most convincing explanation for the observed continua is the composition of scattering and of a single temperature optically thin plasma emission. Spectra and best-fit models are shown in Figure 11 and 2 , respectively, while a summary of the best-fit parameters is reported in Table 2.

\section{$1.2 \quad$ Discussion}

The continuum in the objects of our sample can be deconvolved in at least three separate components (which are likely to be produced in physically and spatially different regions): a) Compton-reflection from neutral or mildly ionized matter (and the associated fluorescent iron line); b) scattering of a Seyfert 1like primary continuum (and the associated fluorescent ionized emission lines); c) optically thin thermal plasma.

The most likely origin for the Compton reflection is the matter responsible for the complete obscuration of the nuclear continuum (e.g.: the molecular torus envisaged by the unification theories). High-resolution imaging in the optical (Malkan et al. 1997) and IR (Granato et al. 1997; Maiolino et al. 1998) showed that the distribution of matter on the scale of tens of pc is very complex, with dusty lanes or bars protruding towards the center, which might as well contribute to the X-ray extinction.

In the case of Circinus Galaxy, the $2.8 \mathrm{keV}$ EW iron line, coupled with the relatively low inclination angle 
Table 2: Continuum parameters for NGC 1068 and Circinus Galaxy best-fit models

\begin{tabular}{lcc}
\hline \hline & NGC 1068 & Circinus Galaxy \\
$\mathrm{N}_{\mathrm{H}}\left(10^{20} \mathrm{~cm}^{-2}\right)$ & $3.1 \pm 0.3$ & $80 \pm_{50}^{60}$ \\
$\Gamma$ & $2.13 \pm 0.17$ & $1.6 \pm_{0.3}^{0.2}$ \\
$\mathrm{~F}_{\text {Compton }}^{\ddagger}\left(10^{-12} \mathrm{erg} \mathrm{cm}^{-2} \mathrm{~s}^{-1}\right)$ & 1.9 & 6.3 \\
$\mathrm{~F}_{\text {scattering }}^{\ddagger}\left(10^{-12} \mathrm{erg} \mathrm{cm}^{-2} \mathrm{~s}^{-1}\right)$ & 2.2 & 3.0 \\
$\mathrm{~L}_{\text {th }}\left(10^{41} \mathrm{erg} \mathrm{s}^{-1}\right)^{\dagger}$ & 3.7 & 0.14 \\
$\mathrm{kT}_{\text {th }}$ & $440 \pm 50$ & $500 \pm_{300}^{700}$ \\
$\mathrm{~A}_{\mathrm{Z}}(\%)$ & $2.8 \pm 1.6$ & $23 \pm_{22}^{465}$ \\
$\chi_{\nu}^{2}$ & 1.02 & 0.86 \\
\hline \hline
\end{tabular}

¥ in the $2-10 \mathrm{keV}$ band $\quad \dagger^{i n}$ the $0.5-4.5 \mathrm{keV}$ band

derived from the hard X-ray analysis (Matt et al. 1999), implies an iron overabundance by at least a factor of 3 (Matt et al. 1996a; Matt et al. 1997b). In NGC 1068 the situation is far more complex. If we assume the iron line deconvolution in Table 1, the EW of the neutral component is $\lesssim 1.6 \mathrm{keV}$, suggesting a high inclination. This is in agreement with the idea that the line comes from the same region as the water maser $\left(\iota \gtrsim 82^{\circ}\right.$, Greenhill et al. 1996).

The warm scattered continuum is described as a fainter replica of the intrinsic nuclear power-law. If the spectral indices of the primary and scattered continuum are left independently free to vary in the fit, no significant difference is found (in NGC 1068: $\Delta \Gamma \equiv \Gamma_{\text {primary }}-\Gamma_{\text {scattered }}=0.3 \pm_{0.5}^{0.4}$; in Circinus Galaxy, $\left.\Delta \Gamma=0.1 \pm_{1.0}^{1.6}\right)$. The bulk of the ionized lines observed in the X-ray spectra of both targets is likely to originate as resonant scattering or fluorescence in the same medium. In Circinus Galaxy the lack of ionized iron emission lines is consistent with a single-zone scatterer with intermediate ionization parameter. On the other hand, in NGC 1068 the wide range of elements involved (and, consequently, of ionization stages required to support the existence of different elements in He- or H-like stages) implies that the reflector need not to be one-zone or homogeneous. The most likely configuration is a continuum distribution of ionization parameters $\xi$ in the whole range $10^{2}-10^{4}$ (Netzer et al. 1997). Following Matt et al. (1996a), we used the ratio of the Compton-reflected (which depends mainly on the inclination angle) with the warm scattered flux (which depends almost entirely on the optical depth of the scattering material) to derive an estimate of the column density of the warm mirror: $\lesssim$ a few $10^{21} \mathrm{~cm}^{-2}$ in NGC 1068 and a few $10^{22} \mathrm{~cm}^{-2}$ in Circinus Galaxy.

A $\mathrm{N}_{\mathrm{H} \text {,warm }} \sim 10^{22} \mathrm{~cm}^{-2}$ scatterer should also imprint absorption feature in the soft X-ray spectrum. The addition of photoionization absorption edges, however, does not yield any improvement in the quality of the fit. The upper limits on the optical depths of OvII and OvIII photoionization edges are 0.35 and 0.25 in NGC 1068, respectively. The same limits are 2.7 and 1.5 in the low Galactic latitude (and therefore highly absorbed) Circinus Galaxy. Assuming that the scattering plasma is in the typical conditions of the "warm absorbers" observed in Seyfert 1 galaxies (Reynolds 1997; George et al. 1998), this corresponds to hydrogen equivalent column densities of $\mathrm{N}_{\mathrm{H} \text {,warm }} \lesssim 5 \times 10^{21} \mathrm{~cm}^{-2}$ and $\mathrm{N}_{\mathrm{H} \text {,warm }} \lesssim 4 \times 10^{22} \mathrm{~cm}^{-2}$, broadly in agreement with the above diagnostics. More conclusive statements have to be deferred to future high-resolution soft X-ray spectroscopical data.

We tentatively associate the thermal component with the contribution of the intense nuclear starburst. David et al. (1992) derived an empirical correlation between the FIR and the X-ray luminosity of starbursts. If we apply their scale law, the expected X-ray luminosities are $\simeq 10^{41} \mathrm{erg} \mathrm{s}^{-1}$ and $\simeq$ $2.1 \times 10^{40} \mathrm{erg} \mathrm{s}^{-1}$ for NGC 1068 and Circinus Galaxy, respectively, broadly consistent with the observed ones, given also the admittedly strong uncertainties on the correlation parameters. This strengthens the case for our identification. A breakthrough in this field will be provided by space-resolved spectroscopy 


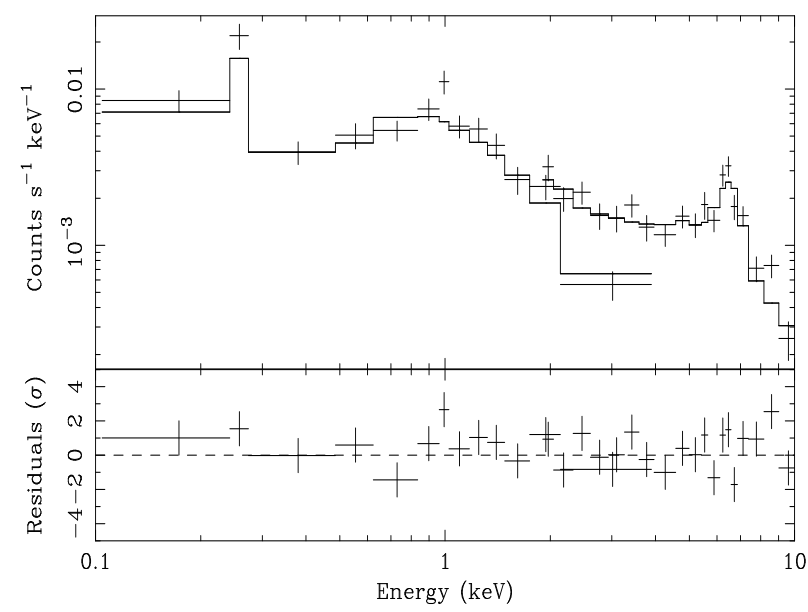

Figure 3: Spectra (upper panels) and residuals in units of standard deviations (lower panels), when the broadband BeppoSAX spectrum is fitted with a simple power-law + "bare reflection" model

on arc seconds scale, which the forthcoming Chandra high-resolution detectors will allow.

\section{SOFT X-RAYS IN THE OFF STATE OF THE SEYFERT 1 GALAXY NGC 4051}

The strongly X-ray variable (Lawrence et al. 1987; Matusoka et al. 1990; Guainazzi et al. 1996; Uttley et al. 1998) and low-luminosity Seyfert 1 galaxy NGC 4051 was observed by BeppoSAX on May 1998 in an ultra-low and stable state, which was best explained assuming that the active nucleus had switched off, leaving only a residual reflection component visible (Guainazzi et al. 1998). A similar flux decrease was observed only in the "fading" Seyfert 2 NGC 2992 (Weaver et al. 1992). A prominent soft excess above the extrapolation of the hard X-ray best-fit is evident below $4 \mathrm{keV}$. This soft component cannot be unambiguously modeled. A power-law, an optically thin plasma, or a double blackbody provide comparably good fits, as shown in Table 3 and Figure 2, while a scattering scenario alone is ruled out. The $0.5-2 \mathrm{keV}(0.1-2 \mathrm{keV})$ observed flux is $\simeq 7.4(12.1) \times 10^{-13} \mathrm{erg} \mathrm{cm}^{-2} \mathrm{~s}^{-1}$, corresponding to an

\begin{tabular}{lccccc}
\hline \hline Soft excess model & $\begin{array}{c}\mathrm{N}_{\mathrm{H}} \\
\left(10^{20} \mathrm{~cm}^{-2}\right)\end{array}$ & $\Gamma_{\text {hard }}$ & $\begin{array}{c}\Gamma_{\text {soft }} \text { or } \mathrm{kT}_{\mathrm{bb}}^{1} \text { or } \mathrm{kT}_{\text {mekal }} \\
(\mathrm{keV})\end{array}$ & $\begin{array}{c}\mathrm{N}_{\text {soft }} / \mathrm{N}_{\text {hard }} \text { or } \mathrm{kT}_{\text {bb }}^{2} \text { or } \mathrm{Z}_{\text {mekal }} \\
(\mathrm{keV}) \text { or }(\%)\end{array}$ & $\begin{array}{c}\chi^{2} / \mathrm{dof} \\
\text { or }\end{array}$ \\
\hline Power-law & $3.4 \pm_{1.0}^{1.2}$ & $1.75 \pm_{0.15}^{0.18}$ & $3.0 \pm_{0.3}^{0.2}$ & $13 \pm_{6}^{13}$ & $<16.2 / 93$ \\
mekal & $1.18^{\ddagger}$ & $1.92 \pm_{0.13}^{0.19}$ & $0.75 \pm_{0.10}^{0.09}$ & $<1.5$ & $111.6 / 92$ \\
Double blackbody & $<2.0$ & $1.98 \pm_{0.16}^{0.19}$ & $0.31 \pm_{0.04}^{0.05}$ & $0.095 \pm_{0.012}^{0.014}$ & $115.9 / 81$ \\
\hline \hline
\end{tabular}

$\ddagger$ unconstrained

Table 3: Best-fit parameters for the broadband NGC4051 Beppo-SAX spectrum. Different models in column 1 correspond to different description of the soft excess. $\Gamma_{\text {hard }}$ is the photon index of the nuclear continuum, which gives rise to the Compton reflection component dominating above $4 \mathrm{keV}$

unabsorbed rest frame luminosity of $1.7(7.0) \times 10^{40} \mathrm{erg} \mathrm{s}^{-1}$. There is no evidence of absorption edges and/or emission lines, the $90 \%$ upper limit on the optical depth of O VII and O VIII photoabsorption edges being 0.34 and 0.13 , respectively. The steepness of the spectrum and the lack of any "warm absorber" imprinting, strongly point against a nuclear origin of the soft $\mathrm{X}$-rays. The intrinsic $0.1-2 \mathrm{keV}$ luminosity inferred by the present data is high but not uncommon for a "normal" galaxy (Fabbiano 1989). It is therefore possible that the dimming of the nucleus has left the galactic components (supernovae winds, hot halos) as the bulk of the observed soft X-rays. Alternatively, the observed soft X-rays could have the 
same origin as the extended (spatial scale $\sim 100 \mathrm{pc}$ ) emission observed by the ROSAT HRI in NGC 4151 (Morse et al. 1995), which coincides with the optical narrow line emitting clouds. It was interpreted as thermal emission from a hot $\left(\mathrm{T} \sim 10^{7} \mathrm{~K}\right)$ and low density $\left(\mathrm{n}_{\mathrm{e}}<1 \mathrm{~cm}^{-3}\right)$ gas, in pressure equilibrium with the Narrow Line Region clouds. Again, space-resolved spectroscopy would be invaluable under this respect, if the NGC 4051 will be so kind to let herself to be caught again in a ultra-dim state.

\section{REFERENCES}

Antonucci R., ARA\&A, 31, 473 (1993)

Antonucci R. \& Miller J.S., ApJ, 297, 621 (1985)

Awaki H., Koyama K., Inoue H., Halpern J.P., 43, 195 (1991)

Boella G., Butler R., Perola G.C., A\&AS, 112, 299 (1997)

David L.P., Jones C., Forman W., ApJ, 388, 82 (1992)

Fabbiano G., ARA\&A, 27, 87 (1989)

George I.M., et al., ApJS, 114, 73 (1998)

Granato G.L., Danese L., Franceschini A., ApJ, 486, 147 (1997)

Greenhill L.J., et al., ApJ, 472, L21 (1996)

Guainazzi M., et al., MNRAS, 301, L1 (1998)

Guainazzi M., et al., MNRAS, submitted (1999)

Guainazzi M., Mihara T., Otani C., Matsuoka M., PASJ, 48, 781 (1996)

Heisler C.A., Lumsen S.L., Bailey J.A., Nature, 385, 700 (1997)

Iwasawa K., Fabian A.C., Matt G., MNRAS, 289, 443 (1997)

Lawrence A., Watson M.G., Pounds K.A., Elvis M., Nature, 325, 694 (1987)

Maiolino R., et al., ApJ, 493, 650 (1998)

Matsuoka M., Piro L., Yamauchi M., Murakami M., ApJ, 361, 440 (1990)

Matt G., Brandt W.N., Fabian A.C., MNRAS, 280, 823 (1996a)

Matt G., et al., A\&A, 325, L13 (1997a)

Matt G., et al., A\&A, 341, L39 (1999)

Matt G., et al., MNRAS, 281, 69 (1996b)

Matt G., Fabian A.C., Reynolds C.S., MNRAS, 289, 175 (1997b)

Morse J.A., Wilson A.S., Elvis M., Weaver K.A., ApJ. 439, 121 (1995)

Nandra K., George I.M., Mushotzky R.F., Turner T.J., Yaqoob T., ApJ, 477, 602 (1997)

Netzer H., Turner T.J., George I.M., ApJ, 488, 694 (1997)

Oliva E., Marconi A., Cimatti A., Di Serego Alighieri S., A\&A, 329, L21 (1998)

Ptak A., Serlemitsos P., Yaqoob T., Mushotzky R., ApJS, in press (astroph/9808159, 1999)

Reynolds C.S., MNRAS, 286, 513 (1997)

Scoville N.Z., ApJ, 327, 61 (1988)

Tran H.D., ApJ, 440, 565 (1995)

Turner T.J., George I.M., Nandra K., Mushotzky R.F., ApJS, 113, 23 (1997)

Ueno S., et al., PASJ, 46, L71 (1994)

Uttley P., McHardy I., Papadakis I.E., Cagnoni I., Fruscione A., Nucl. Phys. B., 69/1-3, 490 (1998)

Weaver K.A., et al., ApJ, 458, 160 (1996) 